\title{
Abhandlungen
}

\author{
Thomas Haipeter
}

\section{Arbeits(zeit)politik zwischen Innovation und Eigensinn}

\begin{abstract}
Der vorliegende Beitrag versteht sich als Beitrag zur aktuellen Debatte um Chancen und Widerstände der Arbeitspolitik. Dabei wird die Perspektive auf die Arbeitszeitpolitik als wichtigem Element der Arbeitspolitik ausgeweitet. Ein genauerer empirischer Blick auf die arbeitszeitpolitische Praxis in den Betrieben zeigt, dass neue Arbeitszeitregulierungen mit innovativem arbeitspolitischem Gehalt vielfältigen Umsetzungsproblemen in den Betrieben ausgesetzt sind. Die vielleicht wichtigste Voraussetzung für eine gute arbeitszeitpolitische Praxis im Sinne der Erhöhung der Autonomie der Beschäftigten bei der Arbeitszeitgestaltung ist eine Interessenvertretungspolitik der Betriebsräte, die auf die Förderung der Partizipationsmöglichkeiten der Beschäftigten abzielt. Diese Politik einer Ausweitung der industriellen Bürgerrechte kann zugleich die in der Debatte als Gegensatz eingeführten Konzepte der innovativen und der eigenständigen Arbeitspolitik versöhnen.
\end{abstract}

\section{Einleitung: Arbeitspolitische Debatten}

Arbeitspolitik steht, wie Klaus Dörre zu Recht feststellt (2005), wieder auf der Tagesordnung sozialwissenschaftlicher Kontroversen. Mitte der neunziger Jahre war das Thema zunächst in den Hintergrund getreten. Von Arbeitspolitik war nur noch wenig zu hören, obwohl der aus der angelsächsischen Debatte um den Arbeitsprozess stammende Begriff in den achtziger und frühen neunziger Jahren in der deutschen Diskussion große Aufmerksamkeit gefunden hatte, weil er erstmals die Offenheit und den politischen Charakter arbeitsorganisatorischen Wandels in das Blickfeld der Forschung rückte (dazu Jürgens/Naschold 1983).

Die Diskussion um Arbeitspolitik war zunächst unlösbar mit der Perspektive einer Überwindung des Taylorismus in der Arbeitsorganisation verbunden. Diese Perspektive wurde vor kurzem im Konzept der „innovativen Arbeitspolitik“ systematisch ausgearbeitet. Das Konzept gründet sich auf die Annahme, dass sich in der Arbeitspolitik bessere Arbeitsbedingungen für die Beschäftigten im Sinne erhöhter Autonomiespielräume und erweiterter Aufgabenzuschnitte auf der einen und höhere Wirtschaftlichkeit für die Unternehmen auf der anderen Seite zu einem Positivsummenspiel verbinden lassen. Als wichtige Voraussetzung dafür wird ein kohärentes Verhältnis zu den betrieblichen Rahmenbedingungen wie Betriebsorganisation, Führungs- oder Entgeltstrukturen benannt (Kuhlmann/Sperling/Balzert 2004, $39 \mathrm{ff})$.

Arbeit, Heft 2, Jg 15 (2006), S. 73-84 
Das Konzept der innovativen Arbeitspolitik ist in der arbeitspolitischen Debatte mit zwei Argumenten kritisiert worden (so Sauer 2005; Kratzer/Sauer 2003). Das erste Argument lautet, dass die in der innovativen Arbeitspolitik angelegte Kritik am Taylorismus als Referenzfolie den aktuellen Entwicklungen der Reorganisation von Arbeit nicht mehr Genüge leisten könne. In vielen Unternehmen hätten neue Organisationsformen Platz gegriffen, die zwar eine Überwindung des Taylorismus bedeuteten, die aber nicht zu einer Verbesserung der Arbeitsbedingungen geführt hätten. Die zunehmende Nutzung des Marktes als Steuerungsinstrument der Organisation und ein damit verbundener neuartiger Zugriff auf die subjektiven Potenziale der Beschäftigten führe vielmehr dazu, dass der mögliche Zugewinn an Autonomie mit einer erweiterten Nutzung der Arbeitskraft einhergeht, die im Nettoeffekt eine Verbesserung der Arbeitsbedingungen nicht erwarten lässt.

Das zweite Argument lautet, dass es unter den Bedingungen „,vermarktlichter“ Organisationen keine Grundlagen mehr für Positivsummenspiele gäbe, weil die an die Beschäftigten herangetragenen Anforderungen des Marktes maßlos seien und man mit dem Markt nicht verhandeln könne (Sauer 2005, 183). Die für eine innovative Arbeitspolitik notwendigen kooperativen Kompromissstrukturen hätten deshalb vielfach aufgehört zu existieren. Daraus wird der Bedarf an einer ,eigensinnigen Arbeitspolitik“ abgeleitet, die in Abgrenzung zur innovativen Arbeitspolitik nicht mehr auf die Interessen der Unternehmen Bezug nimmt, sondern sich direkt aus den autonomen Interessen der Arbeitskraft zu begründen habe.

Damit steht die Diskussion um Arbeitspolitik vor der entscheidenden Frage, ob sich der mit dem Konzept ursprünglich verbundene optimistische Bezugsrahmen für die Gestaltung der Arbeit prinzipiell noch aufrecht erhalten lässt oder ob das Konzept lediglich noch Defensivpositionen beschreiben kann. Diese Frage möchte ich im Folgenden am Beispiel der Arbeitszeitpolitik und der Arbeitszeitregulierung behandeln. Zum einen existieren zwischen neuen Formen der Arbeitszeitregulierung und einer innovativen Arbeitspolitik bedeutsame inhaltliche Überschneidungen. Und zum anderen fanden in den letzten Jahren gerade in der Arbeitszeitpolitik verbreitet arbeitspolitische Veränderungen statt. Dabei hat sich gezeigt, dass Ansatzpunkte für eine innovative Arbeitspolitik auch unter den Bedingungen marktorientierter Steuerungsformen existieren. Die Durchsetzung dieser Politik ist aber an eine Interessenvertretungspolitik der Betriebsräte gebunden, in deren Zentrum die Förderung der individuellen Beteiligungsrechte der Beschäftigten steht. Eine solche Politik enthält zugleich Elemente einer eigensinnigen Arbeitspolitik, die über den engeren Rahmen betriebswirtschaftlicher Verträglichkeit hinausweist. Innovative und eigensinnige Arbeitspolitik sind daher nicht als Gegensätze zu verstehen, sondern können sich wechselseitig ergänzen.

\section{Arbeitszeitregulierung als Element einer innovativen Arbeitspolitik}

Spätestens seit Anfang der neunziger Jahre gehören die Flexibilisierung der Arbeitszeiten und der Übergang zur „atmenden Produktion“ (Hartz 1996) zu den zentralen Elementen der Reorganisation deutscher Unternehmen. Inzwischen arbeiten mehr als 40\% der Beschäftigten mit Arbeitszeitkonten, die als wichtigstes Instrument der Flexibilisierung der Arbeitszeiten betrachtet werden können (Bauer u.a. 2004). Arbeitszeitkonten sind in hohem Maße Gegenstand betrieblicher Regulierungen. Als „,regulierte Flexibilität“ stellen sie eine neue Form der Arbeitszeitregulierung dar (Seifert 2001; Haipeter/Lehndorff 2002). Diese neue Form beruht 
auf der „Verbetrieblichung“ der Regulierungsebenen (Schmidt/Trinczek 1999, 120). Durch die Festlegung von Ausgleichszeiträumen für Schwankungen der Regelarbeitszeit wurden in den Flächentarifverträgen Spielräume für unterschiedliche Verteilungen der Regelarbeitszeiten geschaffen, deren konkrete Ausgestaltung in die Hoheit der Betriebsparteien verlagert wurde.

Die betrieblichen Akteure nehmen aber eine neue Rolle nicht nur als Gestalter der Arbeitszeitnormen, sondern auch als Akteure der Arbeitszeitpraxis ein. Denn mit den neuen Regulierungsformen ist häufig eine Neufassung der Handlungsnormen und -möglichkeiten von Betriebsräten und Beschäftigten verbunden. Mit Blick auf die Betriebsräte existieren vielfältige neue Prozessnormen der Mitbestimmung, die beispielsweise darin bestehen, dass Betriebsräte bei Überschreitung bestimmter Saldengrenzen in Aushandlungen mit dem Management über Rückführungsmöglichkeiten der Arbeitszeiten oder über Personalanpassungen treten oder dass die Beschäftigten die Betriebsräte bei Problemen ihrer Arbeitszeitgestaltung als Interessenvertreter hinzuziehen können. Mit Blick auf die Beschäftigten ist eine neuartige - und wie weit auch immer in der konkreten Regulierung gefasste - Handlungsautonomie bei der Gestaltung ihrer eigenen Arbeitszeit festzustellen. Die Beschäftigten werden in der neuen Arbeitszeitregulierung zu Subjekten der Arbeitszeitgestaltung im Betrieb.

Diese Begründung einer Handlungsautonomie der Beschäftigten in der Arbeitszeitgestaltung markiert die zentrale Schnittstelle zwischen den neuen Formen der Arbeitszeitregulierung und dem Konzept der innovativen Arbeitspolitik. Die Autonomiespielräume, die den Beschäftigten bei der Gestaltung ihrer Arbeitszeit zuerkannt werden können, sind ein wichtiges Element der Verbesserung ihrer Arbeitsbedingungen. Anders als früher können die Beschäftigten nunmehr selber die Entscheidung darüber fällen, zu welchen Zeitpunkten und in welcher Länge sie täglich und verteilt über bestimmte Zeiträume arbeiten. Damit werden Möglichkeiten für die Vereinbarung arbeitsbezogener und lebensweltlicher Zeitinteressen geschaffen. Diese Möglichkeiten sind zumeist mit Normen für die Partizipation der Beschäftigten in der Arbeitszeitgestaltung verbunden. Im Unterschied zur auf das individuelle Arbeitszeithandeln bezogenen Autonomie verstehe ich Partizipation als Beteiligung der Beschäftigten an der Gestaltung ihrer Arbeitsbedingungen in Aushandlungen mit ihren Führungskräften. Autonomie und Partizipation stehen in engem Zusammenhang. Verbesserungen der Autonomie sind insbesondere dann zu erwarten, wenn es den Beschäftigten in Aushandlungen gelingt, Einfluss auf Rahmenbedingungen zu nehmen, die für mögliche Restriktionen ihrer Autonomie verantwortlich sind, wenn sie also „Handlungsautonomie“ durch „Verhandlungsautonomie“ ergänzen können (Moldaschl 2001, 136).

Die neuen Formen der Arbeitszeitregulierung können auch zu einer Erhöhung der Wirtschaftlichkeit für die Unternehmen beitragen, und zwar aus zwei Gründen. Der erste Grund ist der, dass die Beschäftigten selbständig dazu übergehen, ihre Arbeitszeiten an die betrieblichen Erfordernisse anzupassen. Auf diese Weise kann eine bessere Deckung von Arbeitseinsatz und Arbeitsanfall erzielt werden, als dies allein durch kollektive Entscheidungen der Betriebsparteien möglich wäre. Zweitens kann der Beschäftigte auf dieser Grundlage für seine Entscheidung zur Verantwortung gezogen werden. Bei Überschreitungen der Zeitgrenzen kann so die Frage aufgeworfen werden, wofür der Beschäftigte eigentlich seine Arbeitszeit verwendet und wie er in seiner Arbeitszeit gearbeitet hat. Auf dieser Grundlage können sowohl die Effektivität und Effizienz der Einzeltätigkeiten verbessert als auch Probleme der organisatorischen Rahmenbedingungen aufgedeckt werden.

Die Frage der Anknüpfungspunkte zwischen Arbeitszeitregulierung und innovativer 
Arbeitspolitik lässt sich noch weiterführen. Dies wird deutlich, wenn der Aspekt der Kohärenz der Arbeitspolitik zu anderen Variablen der Betriebsorganisation betrachtet wird. Arbeitszeitregulierung kann die Kohärenz der betrieblichen Reorganisation in Richtung einer innovativen Arbeitspolitik deutlich stärken. Das möchte ich kurz an drei Punkten zeigen. Der erste Punkt betrifft den Einfluss auf die betriebliche Führung. In den Arbeitszeitregulierungen erhalten die Beschäftigten Partizipationsrechte. Die damit verbundenen diskursiven Problemlösungsverfahren geben den Führungskräften normative Anreize für die Entwicklung dialogischer Führungsstile. Zweitens können die Autonomiespielräume der Beschäftigten in der Arbeitsorganisation durch die Arbeitszeitregulierung institutionell abgesichert werden. Auf diese Weise kann entweder eine Neuausrichtung der Arbeitsorganisation angestoßen oder bereits vorhandene innovative Ansätze können gestärkt werden. Drittens könnten im Bereich der Entgeltsysteme Ansätze der innovativen Arbeitspolitik wie Zielvereinbarungssysteme unterstützt werden, die eine Mitsprache der Beschäftigten bei der Ermittlung von Leistungszielen vorsehen. Nicht nur werden damit, wie bei der Arbeitszeitregulierung, Normen für dezentrale Aushandlungen zwischen Beschäftigten und ihren Führungskräften geschaffen, sondern diese kreisen inhaltlich auch um die gemeinsame Frage nach dem Verhältnis von Arbeitszeit und Arbeitsleistung.

Arbeitszeitregulierung kann daher als Kernelement einer innovativen Arbeitspolitik betrachtet werden. Die entscheidende Frage lautet allerdings, ob die Arbeitszeitregulierung auch tatsächlich in den Betrieben in dieser innovativen Art und Weise funktioniert. In der „doppelten Wirklichkeit“ von Organisationen (Weltz 1988) fallen formale Strukturen und tatsächliche Handlungen häufig auseinander. Die betriebliche Praxis kann sich ziemlich unabhängig von den Vorgaben formaler Regelungen entwickeln. Und tatsächlich weisen empirische Befunde darauf hin, dass es in der betrieblichen Praxis einige Probleme der Wirksamkeit neuer Arbeitszeitnormen gibt.

\section{$3 \quad$ Funktionsprobleme der flexiblen Arbeitszeitregulierung}

Im Rahmen eines von der HBS finanzierten Forschungsprojektes hatten wir uns jüngst mit der Wirkungsweise neuer Formen der Arbeitszeitregulierung in der betrieblichen Praxis beschäftigt und nach den Erfolgsbedingungen für eine hohe Bindekraft der Arbeitszeitnormen gefragt (dazu auch Haipeter/Lehndorff 2004). Die Untersuchung beruhte auf fünf Fallstudien mit jeweils 4-7 leitfadengestützten Interviews mit Experten von Management und Betriebsrat sowie jeweils 12-22 Beschäftigteninterviews. Die Fälle waren über verschiedene Branchen und Beschäftigtengruppen gestreut (vom Produktionsbetrieb zur IT-Softwareproduktion, vom Produktionsarbeiter zum Entwicklungsingenieur). Sie zeichneten sich gemeinsam dadurch aus, dass sie besonders weit entwickelte Formen neuer Arbeitszeitregulierungen darstellten, die ausgefeilte Prozessnormen mit mehreren Kontentypen verbanden. Dabei sind wir auf einige gemeinsame Problembereiche der Regulierungspraxis gestoßen.

Ein erster Problembereich ist die Funktionsweise der Gleitzeitkonten. Zunächst ist festzuhalten, dass in den Interviews fast durchgängig die gute Funktionsfähigkeit der Gleitzeitkonten betont wurde. Die Beschäftigten gaben an, dass sie damit ihre individuellen Zeitinteressen sehr gut zur Geltung bringen könnten. Dabei ist ihnen die Anpassung ihrer Arbeitszeiten an betriebliche Bedarfe - den Arbeitsanfall - zu einer Selbstverständlichkeit geworden. Die betrieblichen Bedarfe sind der Ausgangspunkt, an den sie ihre lebensweltli- 
chen Zeitinteressen anzulagern versuchen. Das Problem der Gleitzeitkonten besteht darin, dass die Beschäftigten auf ihren Konten in der Regel hohe Sockelbestände an Arbeitszeitsalden angehäuft haben, die teilweise weit über das hinausgingen, was in den Arbeitszeitregulierungen als Obergrenzen vorgesehen war. Diese Sockelbestände sind ein Indikator für strukturelle Mehrarbeit, die von den Beschäftigten nach eigener Aussage nicht abbaubar ist und die das gute Funktionieren der Konten langfristig zu gefährden droht.

Daran knüpft die Nutzung der Langzeitkonten als zweites Problem an. Langzeitkonten sind in den Unternehmen als Lösung für das Problem der strukturellen Mehrarbeit eingeführt worden, werden dazu von den Beschäftigten aber kaum eingesetzt. In allen untersuchten Unternehmen strahlten die Langzeitkonten keine hohe Attraktivität für die Beschäftigten aus. Der Hauptgrund dafür lag darin, dass die Beschäftigten nicht darauf vertraut haben, dass die mit den Konten eröffnete Möglichkeit der Entnahme größerer angesparter Zeitblöcke tatsächlich umsetzbar wäre. In der Tat konnte uns in keinem Unternehmen von einem erfolgreichen Fall eines Sabbaticals berichtet werden. Unter diesen Bedingungen reduzierte sich für die Beschäftigten die Funktion der Langzeitkonten auf das Ansparen für die Rente oder für den Vorruhestand. Der Grund für dieses Problem wurde eindeutig benannt: Die knappe Personaldecke, die in den Unternehmen mehr und mehr zur Selbstverständlichkeit geworden ist und die verhindert, dass sich die Beschäftigten bei längeren Zeitentnahmen wechselseitig vertreten können. Und genau darin ist auch die Ursache für die strukturelle Mehrarbeit auf den Gleitzeitkonten zu suchen.

Das dritte Problem ist der Verfall von Arbeitszeiten. Dieses Problem fand sich zwar in unserem Sample nur in einem Fall, spielt nach eigenen weiteren Untersuchungen und der Lage der Literatur aber in Bereichen mit höher qualifizierten Angestelltentätigkeiten eine systematische Rolle (Glißmann/Peters 2001; Haipeter/Lehndorff 2002). Eine wichtige Bedingung dafür besteht darin, dass für die Beschäftigten die Arbeitszeit zu einer eher nebensächlichen Größe geworden ist. Prioritär für die Beschäftigten sind die Anforderungen der Kunden und die Bewältigung des eigenen Arbeitsvolumens. Die Arbeitszeit wird zu einer abhängigen Variablen. Sie gerät erst dann wieder in den Blickpunkt des Interesses, falls gegen die Normen der betrieblichen Regulierung oder des Arbeitszeitgesetzes verstoßen wird. Diese Normen gelten den Beschäftigten als Barrieren bei der Bewältigung ihrer Arbeitsanforderungen, weshalb sie häufig dazu übergehen, ihre Arbeitszeiten nicht mehr exakt zu erfassen. Die Folge sind nicht nur intransparente und unbezahlte, sondern auch extrem lange Arbeitszeiten.

Das vierte Problem schließlich bezieht sich auf die Partizipation der Beschäftigten. Dezentrale Aushandlungen zwischen Beschäftigten und Führungskräften fanden in unseren Fällen kaum statt. Die Prozessnormen werden nicht gelebt, weil entweder die Beschäftigten in Problemsituationen ihre Führungskräfte nicht konsultieren, oder weil die Führungskräfte sich aus der Arbeitszeitregulierung zurückziehen, oder weil die Führungskräfte in Problemsituationen mit Anweisungen reagieren und dialogische Verfahren deshalb ins Leere laufen. Im ersten Fall wollen die Beschäftigten ihren zeitlichen Mehraufwand nicht angeben, weil sie ihn als Zeichen persönlichen Versagens werten. Im zweiten Fall lehnen die Führungskräfte die Verantwortung für die Arbeitszeiten mit dem Hinweis ab, dass die Beschäftigten sich ihre Arbeitszeiten selber zugewiesen hätten. Und im dritten Fall ignorieren die Vorgesetzten ihre neuen Führungsrollen und ziehen die Gestaltung der Arbeitszeiten an sich. Diese Probleme der Partizipation sind für die innovative Arbeitspolitik von entscheidender Bedeutung, weil damit den Beschäftigten verwehrt wird, auf Ursachen für Probleme ihrer autonomen Arbeitszeitgestaltung Einfluß zu nehmen, die in den Rahmenbedingungen ihrer Arbeit liegen. 


\section{$4 \quad$ Typen der Partizipationspraxis}

Unsere betrieblichen Fälle haben sich hinsichtlich der praktischen Probleme der Partizipation erheblich unterschieden. Diese Unterschiede lassen sich analytisch generalisieren (dazu Yin 1988, 27ff) und zu drei Typen der Partizipationspraxis in den Unternehmen bündeln. Von den drei Typen sind zwei als defizitär mit Bezug auf die praktische Wirksamkeit der Prozessnormen zu bewerten, der dritte Typ hingegen kann als Variante einer guten Regelungspraxis bezeichnet werden (dazu auch Haipeter 2005).

Der erste Typus ist die Rückkehr der Hierarchie. In diesem Typus wurden Partizipationsnormen der Arbeitszeitregulierung von den unteren Führungskräften für weitgehend unwirksam erklärt. Dieser Fall war typisch für Bereiche der direkten Produktion in Industrieunternehmen, in denen die Vorgesetzten selber strengen Leistungszielen unterworfen waren. Hier ließ sich feststellen, dass die Vorgesetzten die mit der Arbeitszeitregulierung verbundenen Spielräume der Arbeitszeitgestaltung unter dem Eindruck wachsenden Kostendrucks zurückgenommen haben. Damit konnte sich ein echter Dialog über die Verwendung von Arbeitszeiten oder die Rückführung von Arbeitszeiten nicht entfalten. Allerdings haben die Vorgesetzten sich dabei weitgehend an die normativen Vorgaben zu Verteilung und Länge der Arbeitszeiten gehalten.

Im zweiten Typus, der Dominanz der Marktsteuerung, ist das Problem gegensätzlich gelagert. Dieser Typus findet sich in Fällen hoch qualifizierter Angestelltenarbeit, in denen Subjektivierung und Selbstorganisation zu selbstverständlichen Tatbeständen der Unternehmensorganisation und-kultur gehören. In diesen Bereichen ist zwar die autonome Gestaltung der Arbeitszeit inzwischen gängige Praxis. Doch kann diese Praxis damit verbunden sein, dass sich die Vorgesetzten aus der Arbeitszeitregulierung weitgehend zurückziehen mit der Begründung, dass die Beschäftigen selbst für die Einhaltung der Arbeitszeitnormen und die Rückführung überschüssiger Arbeitszeiten zu sorgen hätten. Auch in dieser Situation finden die partizipativen Verfahren keine Ansatzpunkte in der sozialen Praxis der Betriebe. Diese Situation ist in der Regel mit überlangen Arbeitszeiten verbunden.

Der dritte Typus, die regulierte Marktsteuerung, konnte in einem der Fallunternehmen beobachtet werden und steht für eine gute Partizipationspraxis. Auch er ist in der hoch qualifizierten Angestelltenarbeit angesiedelt. Die autonome Organisation der Arbeitszeit ist wie selbstverständlich Sache der Beschäftigten. Im Unterschied zum vorigen Typus jedoch spielen die Vorgesetzten eine aktive Rolle bei der Umsetzung der partizipativen Verfahren der Arbeitszeitregulierung. So entscheiden Vorgesetzte und Beschäftigte gemeinsam darüber, ob beispielsweise ein Mehrarbeitsbedarf vorliegt und deshalb ein Zusatzvolumen an Arbeitszeit zu vereinbaren ist. Und in den Arbeitszeitgesprächen wird danach gefragt, welche Funktionen und Tätigkeitsfelder eines Projektes wirklich wichtig sind. Auf diese Weise werden Ansatzpunkte zum Lernen in der Organisation geschaffen, und, mehr noch, die Rahmenbedingungen thematisiert, unter denen die Beschäftigten ihre Arbeitszeiten gestalten.

\section{$5 \quad$ Marktorientierte Steuerungsformen und ihre Folgen}

Die drei Partizipationstypen sind Stilisierungen einer Vielfalt der Arbeitszeitpraxis in Unternehmen mit flexiblen Arbeitzeitregulierungen, die zunächst wenig gemein zu haben scheinen. 
Doch weisen unsere Fälle eine wichtige Übereinstimmung auf. Alle Unternehmen folgen einem gemeinsamen Pfad der Reorganisation, der sich im Begriff der „marktorientierten Steuerungsformen“ oder des „marktzentrierten Kontrollmodus“ (Dörre 2001) bündeln lässt. Die Unternehmen nutzen Marktmechanismen zur Steuerung interner Prozesse. Dies geschieht in zwei sich ergänzenden Formen.

Die erste Form ist die „Finanzialisierung“ der Corporate Governance. Sie zeichnet sich dadurch aus, dass Normen und Erwartungen von Finanzmarktakteuren zur Legitimierung strategischer Entscheidungen herangezogen werden (Kädtler 2003). Gestützt durch einen Wandel der institutionellen Rahmenbedingungen - zu dem der Bedeutungsanstieg der Finanzmärkte ebenso gehört wie der Rückzug der Großbanken aus der Unternehmensfinanzierung und -kontrolle oder der Wandel der Entlohnung und des Sozialprofils von Top Managern (Höpner 2003; Jackson 2003) - findet in den Unternehmen eine Konzentration auf kurzfristige Renditeziele statt. Diese werden mit Hilfe finanzwirtschaftlicher Kennzahlen als Zielvorgaben in die Organisationen eingeführt.

Die zweite Form ist die operative Dezentralisierung. Sie beruht auf der Gründung dezentraler Einheiten, denen die Verantwortung für Kosten und Profite übertragen wird. Die dezentralen Einheiten werden zugleich der Marktkonkurrenz ausgesetzt, und zwar sowohl der externen als auch der internen Konkurrenz. Daraus entsteht eine neuartige Konkurrenz zwischen Produktionsstandorten, deren Leistungsfähigkeit mit Verfahren des Benchmarking verglichen werden kann. Die Erhaltung eines Standortes ist nicht mehr nur die Aufgabe des Managements, sondern wird zum Problem jedes Beschäftigten bei der Sicherung des eigenen Arbeitsplatzes. Diese Konfrontation der Beschäftigten mit dem Markt ist auch als indirekte Steuerung bezeichnet worden (vgl. dazu Glißmann/Peters 2001).

Die Einführung marktorientierter Steuerungsformen scheint auf den ersten Blick in einem positiven Zusammenhang zur flexiblen Arbeitszeitregulierung zu stehen. Flexible Arbeitszeitregulierungen sind eine notwendige Bedingung dafür, dass operative Einheiten, und in diesen Einheiten die Beschäftigten selber, auf die Veränderungen der Marktbedingungen hinreichend passgenau reagieren können. Doch darf darüber nicht übersehen werden, dass marktorientierte Steuerungsformen auch potenziell negative Konsequenzen für die Arbeitszeitregulierung nach sich ziehen können. Dabei stehen vor allem zwei Entwicklungen im Vordergrund.

Erstens ist zu erwarten, dass die Konfrontation sowohl dezentraler operativer Unternehmenseinheiten als auch der Beschäftigten mit den Marktanforderungen Druck auf die praktische Wirksamkeit der Arbeitszeitregulierung ausüben kann. Betriebsräte könnten in der Standortkonkurrenz in die Situation geraten, mit dem Ziel des Erhalts von Standort und Beschäftigung eine Absenkung von Arbeitszeitnormen zuzulassen oder eine faktische Unterschreitung bestehender Normen zu tolerieren. In ähnlicher Weise könnten Beschäftigte versucht sein, zum Erhalt ihres Arbeitsplatzes und zur Bewältigung der Arbeitsanforderungen die Arbeitszeitregelungen in eigenem Antrieb zu unterlaufen.

Zweitens geht nach unseren empirischen Erfahrungen mit der Finanzialisierung eine Verknappung der Personalressourcen einher. Die Unternehmen begeben sich auf den Pfad einer „Personalpolitik der unteren Linie“, die darauf beruht, aus Kostenmotiven das Personal auf ein operatives Minimum zu reduzieren. Die Senkung des Personalstandes bei gleich bleibendem oder steigendem Arbeitsvolumen führt dann zwangsläufig zu einem Druck auf die Arbeitszeiten und die Einhaltung der Arbeitszeitnormen.

Insoweit deckt sich die Analyse durchaus mit der eingangs vorgestellten Kritik am 
Konzept der innovativen Arbeitspolitik. Wie die innovative Arbeitspolitik im Allgemeinen, so scheint sich auch die innovative Arbeitszeitpolitik im Besonderen an den Folgewirkungen marktorientierter Steuerungsformen zu brechen. Doch plausibel wäre diese Annahme nur, wenn sich die betriebliche Arbeitszeitpraxis mehr oder weniger eindeutig in Richtung des Typus der Dominanz der Marktsteuerung bewegen sollte. Dann hätte der Marktdruck in den Unternehmen ein solches Ausmaß erreicht, dass Arbeitspolitik wirkungslos würde. Diese Schlussfolgerung deckt sich aber nicht mit den aufgezeigten empirischen Erfahrungen. Insbesondere die Existenz der regulierten Marktsteuerung, die sich durch wirksame Partizipationsnormen auszeichnet, lässt sich auf diese Weise nicht plausibel erklären.

Wie und unter welchen Bedingungen kann die innovative Arbeitszeitregulierung im Rahmen marktorientierter Steuerungsformen praktische Wirksamkeit entfalten? Eine erste Antwort auf diese Frage ist in den Regulierungen selbst zu suchen. Es scheint unmittelbar einsichtig, dass mit der Eindeutigkeit und der Präzision, in denen Partizipationsnormen in der Arbeitszeitregulierung formuliert werden, die Wahrscheinlichkeit steigt, dass sie für das soziale Handeln im Betrieb Verbindlichkeit erlangen. Doch ist damit nur eine notwendige, nicht aber eine hinreichende Erfolgsbedingung beschrieben. Denn in unserem Sample fanden sich einige Fälle, in denen starken Regulierungen keine entsprechende soziale Praxis folgt.

\section{Betriebsräte als Anker der Arbeitspolitik}

Meine Hypothese lautet, dass der entscheidende Anker für die innovative Arbeitszeitpolitik bei den Betriebsräten und ihrer Interessenvertretungspolitik zu suchen ist. Dieser Aspekt ist in der Diskussion bislang nur wenig berücksichtigt worden. Zwar war im Zusammenhang mit der innovativen Arbeitspolitik auf die Bedeutung einer Einbindung der Betriebsräte in innovative Konzepte hingewiesen worden. Doch lag diese Rolle vor allem in der Ausarbeitung von Betriebsvereinbarungen; für die praktische Umsetzung hingegen galt der Betriebsrat als ein Impulsgeber neben anderen, der auch durch Prozessbegleiter ersetzbar wäre (Kuhlmann/ Sperling/Balzert 2004, 380). Der Blick auf die innovative Arbeitszeitpolitik zeigt hingegen, dass die Betriebsräte gerade in der Umsetzung unverzichtbar und unersetzbar sind. Dies liegt nicht zuletzt daran, dass Betriebsräte naturgemäß ein größeres Interesse an der Wirksamkeit der innovativen Arbeitszeitregulierung haben als die Unternehmen. Unternehmen, das zeigt der Typus der Dominanz der Marktsteuerung, können, jenseits der positiven Wirkung der Regulierung auf die Senkung der Transaktionskosten, gut mit einer unwirksamen Arbeitszeitregulierung leben, insbesondere wenn damit verbunden ist, dass die Arbeitszeit unentgeltlich und über den Regelungsrahmen hinaus verlängert wird.

Ähnlich aber wie bei den Typen der Partizipationspraxis ist auch für die Interessenvertretungspolitik der Betriebsräte eine Vielfalt empirischer Ausprägungen festzustellen, die sich analytisch zu drei Typen generalisieren lassen. Diese sind nicht von ungefähr als Komplemente zu den oben beschriebenen Typen der Partizipation zu verstehen. Im Unterschied zu den gängigen Typenbildungen der Mitbestimmung oder der betrieblichen Interaktionsmuster in der Literatur (so Bosch u.a. 1999; Kotthoff 1994) zeichnen sie sich dadurch aus, dass sie nicht das Ergebnis einer umfassenden Analyse der betrieblichen Sozial- oder Austauschbeziehungen sind, sondern ausschließlich entsprechend der Rolle der Betriebsräte in der innovativen Arbeitszeitregulierung gebildet wurden. Dies zieht neben einem reduzierten Fokus der Typen auch einen Bias zu Betriebsräten mit einer Orientierung am Co-Management nach sich. Denn 
es sind vor allem diese Betriebsräte, die mit dem Management innovative Arbeitszeitregelungen aushandeln. Ist der Betriebsrat wirkungslos, wird das Management kaum bereit sein, in der Arbeitszeitregulierung Partizipationsrechte in einem Umfang zu gewähren, der dazu berechtigen würden, überhaupt von einer innovativen Arbeitszeitregulierung zu sprechen. Ist der Betriebsrat hingegen stark konfliktorientiert, wird er gemeinsamen Modernisierungsprojekten mit dem Management skeptisch gegenüberstehen und möglicherweise innovative Arbeitszeitregulierungen nicht vereinbaren wollen.

Der erste arbeitszeitpolitische Typus ist der stellvertretende Modernisierer. Dieser Typus zeichnet sich dadurch aus, dass die Betriebsräte in Wettbewerbspakten als Promotoren der betrieblichen Modernisierung agieren und dabei einen erheblichen Einfluss auf die Gestaltung der Reorganisation entwickeln können. In der Arbeitszeitpolitik ist damit typischerweise verbunden, dass die Betriebsräte flexible Arbeitszeitregulierungen fördern und - durchaus nicht ohne Erfolg - versuchen, auf die Entwicklung der Arbeitszeit Einfluss zu nehmen. Vor allem deshalb sind hinsichtlich der Dauer der Arbeitszeiten in diesem Typus die Arbeitszeitregulierungen relativ wirksam. Die unmittelbare Beteiligung der Beschäftigten aber spielt keine erkennbare Rolle für die Betriebsräte; vielmehr verstehen sie sich als Stellvertreter des Beschäftigtenhandelns in den kollektiven Aushandlungen mit dem Management. Dementsprechend setzen sie sich allenfalls zurückhaltend für die Umsetzung partizipativer Verfahrensnormen ein.

Der zweite Typus ist der ohnmächtige Kritiker. Vor allem beheimatet in den ITDienstleistungen, funktionieren bei diesem Typus die Vertretungs- und Legitimationsmuster der Stellvertretung nicht. Das Management akzeptiert die prinzipiell zu einem Co-Management bereiten Betriebsräte nicht als Aushandlungspartner, was vor allem daran liegt, dass die Beschäftigten die Betriebsräte nicht als Repräsentanten ihrer Interessen betrachten. Die Beschäftigten unterlaufen die Arbeitszeitnormen unter dem Eindruck von Zielvorgaben und Marktdruck häufig mit der Konsequenz langer Arbeitszeiten und ungenutzter Partizipationsrechte. Pocht dann der Betriebsrat auf die Einhaltung von Regelungen, gerät er in eine Dilemmasituation, weil er sich damit scheinbar gegen die Interessen der Beschäftigten stellt und seine Stellung weiter schwächt.

Der dritte Typus ist der partizipationsorientierte Interessenmanager. Dieser stellt einen Fall guter Praxis dar. Anders als der ohnmächtige Kritiker wird er als ein Promotor der Modernisierung und als Akteur der Arbeitszeitpolitik von seinem Management anerkannt. Und anders als beim stellvertretenden Modernisierer ist die Durchsetzung direkter Beteiligungsrechte der Beschäftigten ein zentrales Element seiner Arbeitspolitik neben der Einhaltung der Normen zur Dauer der Arbeitszeiten. Die Unterstützung der Beschäftigten bei der Wahrnehmung ihrer Interessen in Aushandlungen mit dem Management versteht er als eine seiner wichtigsten Aufgaben. Er fungiert damit als Promotor der innovativen Arbeitspolitik im Betrieb. Dafür handelt er Verfahrensnormen mit dem Management aus, greift in Problemsituationen ein und ist bereit, eigene Mitbestimmungsrechte an die Beschäftigten abzugeben.

Sowohl der stellvertretende Modernisierer als auch der partizipationsorientierte Interessenmanager können erfolgreich für die Einhaltung der vertraglichen Arbeitszeiten sorgen. Beide Typen zielen aber auf sehr verschiedene Legitimationsgrundlagen ihrer Mitbestimmung ab. Im Fall des stellvertretenden Modernisierers rechtfertigt sich die Mitbestimmung vor allem über ihre Beiträge zur einzelwirtschaftlichen Effizienz des Betriebes in den Modernisierungskoalitionen mit dem Management. Im Fall des partizipationsorientierten Interessenmanagers kann dies zwar auch eine Rolle spielen, doch ist der Betriebsrat weit mehr 
als aktiver Garant der Verteidigung und Ausweitung der industriellen Bürgerrechte der Beschäftigten legitimiert. Damit gerät er weniger als der erste Typus in die Gefahr, in Modernisierungsbündnissen an eigenständigem Profil zu verlieren und von den Beschäftigten mit dem Management verwechselt zu werden. Vielmehr kann er durch das von den Erfahrungen der Partizipation geprägte Selbstverständnis der Beschäftigten einen neuen Rückhalt erfahren, der ihn gerade für die Herausforderungen von Standortkonkurrenz und Finanzialisierung besser gerüstet erscheinen lässt.

\section{Arbeitspolitische Schlussfolgerungen}

Arbeitszeitregulierung ist ein wichtiges Element der innovativen Arbeitspolitik. Sie steht dazu nicht nur in einem engen inhaltlichen Zusammenhang, sondern sie kann der innovativen Arbeitspolitik sowohl aufgrund ihrer großen Bedeutung für die betrieblichen Akteure als auch aufgrund der normativen Rahmung, die sie der Autonomie und der Partizipation der Beschäftigten geben kann, wichtige Impulse ihrer Durchsetzung verleihen. Neue Formen der Arbeitszeitregulierung können dazu beitragen, dass eine innovative Arbeitspolitik unter den Bedingungen marktorientierter Steuerungsformen nicht ohne Erfolgsaussichten sein muss.

Dafür aber bedarf es betrieblicher Akteure und Promotoren. Die wichtigste Rolle kommt dabei den Betriebsräten zu, die für die Arbeitszeitregulierung im Betrieb eine unverzichtbare Ankerfunktion ausüben. Betriebsräte, die sich die Sicherung und Ausweitung individueller Partizipationsrechte als wichtiges Ziel ihrer Interessenvertretungspolitik auf ihre Fahnen geschrieben haben, sind die zentralen Promotoren innovativer Arbeitszeitregulierungen im Betrieb. Mehr noch, diese Form der Interessenpolitik stößt bei den Beschäftigten auf breite Zustimmung und kann den Betriebsräten eine neue Grundlage ihrer betrieblichen Legitimation verschaffen, und zwar als Garanten der Förderung industrieller Bürgerrechte im Betrieb.

Spricht dieses Ergebnis auf der einen Seite für die Umsetzungsfähigkeit einer innovativen Arbeitspolitik, so sind damit auf der anderen Seite aber auch Anknüpfungspunkte für die Forderung nach einer eigensinnigen Arbeitspolitik verbunden, die sich ganz an den Interessen der Beschäftigten ausrichten sollte. Denn in der Ausweitung industrieller Bürgerrechte steckt ein Element, das deutlich über in der innovativen Arbeitspolitik angesprochenen Dimensionen der Wirtschaftlichkeit und der Arbeitsverbesserung hinausgeht. Dieses Element ist die Demokratisierung. Demokratische Teilhabe im Betrieb als Wert für die Beschäftigten erfahrbar zu machen, darin könnte ein unverkennbar eigensinniges Element einer innovativen Arbeitszeitregulierung liegen. In diesem Sinne wäre der partizipationsorientierte Interessenmanager als wichtigster Anker der innovativen Arbeitspolitik auch ein tragender Akteur einer eigensinnigen Arbeitspolitik.

Der Übergang zu diesem Typus der Interessenvertretung ist allerdings nicht voluntaristisch als Entscheidungsproblem für Betriebsräte zu deuten, die einfach zwischen verschiedenen Handlungsalternativen zu wählen hätten. Dagegen sprechen zum einen die hohen Komplexitäts- und Lernanforderungen, die mit diesem Politikverständnis verbunden sind. Betriebsräte dieses Typus müssen gleichermaßen als Ordnungsmacht im Betrieb fungieren, die die Umsetzung ihrer Vereinbarungen (wie auch der Tarifverträge) überwacht, als Gestaltungsmacht, die eigene arbeitspolitische Konzepte entwirft und aushandelt, als Gegenmacht, die in der Lage ist, diese auch gegen die Unternehmensleitung durchzusetzen und schließlich als Manager und Wahrer individueller Beschäftigteninteressen - kein leicht zu erwerbendes 
Rollenprofil.

Gegen eine voluntaristische Sichtweise spricht aber auch, dass das Umfeld für Betriebsräte steiniger wird. Dies gilt vor allem für die zu beobachtende Schwächung des Flächentarifvertrages und seine formale oder faktische Öffnung für betriebsbezogene Unterschreitungen tariflicher Mindestnormen. Die mit dem betrieblichen Konzessionsdruck verbundene defensive Übernahme tarifpolitischer Funktionen könnte die offensiven arbeitspolitischen Gestaltungsziele der Betriebsräte untergraben. Die Folge dieser Entwicklung wäre die Aufkündigung jener klassischen Kompromissstrukturen zwischen den Interessengruppen im Betrieb, die mit dem Begriff der „kooperativen Konfliktbewältigung“ belegt worden waren (Weltz 1977). Unter diesen Bedingungen würde der Spielraum für Positivsummenspiele tatsächlich schwinden. Das würde für die Betriebsräte bedeuten, dass sie sich gegenüber dem Management neu positionieren müssten. Eine Politik des konzessionsbereiten Co-Managements der Modernisierung alleine würde den damit verbundenen Herausforderungen nur noch bedingt gerecht werden, weil der Konzessionsspirale in marktorientierten Steuerungsformen nur schwer Grenzen zu setzen sind. Gerade in dieser Situation aber könnte die Einforderung partizipativer Verfahren und ihrer Geltung im Rahmen einer innovativen Arbeitspolitik für die Betriebsräte eine wichtige Rolle bei der Findung und Legitimierung einer eigenständigen Position gegenüber dem Management spielen.

\section{Literatur}

Bauer, Frank u.a. (2004): Arbeitszeit 2003. Arbeitsgestaltung, Arbeitsorganisation und Tätigkeitsprofile. Köln

Bosch, Aida, Peter Ellguth, Rudi Schmidt, Rainer Trinczek(1999): Betriebliches Interessenhandeln. Band 1: Zur politischen Kultur der Austauschbeziehungen zwischen Management und Betriebsrat in der westdeutschen Industrie. Opladen

Dörre, Klaus (2005): Prekarität-Eine arbeitspolitische Herausforderung; in: WSI Mitteilungen 58, 5, 250258

Dörre, Klaus, (2001): Das deutsche Produktionsmodell unter dem Druck des Shareholder Value; in: Kölner Zeitschrift für Soziologie und Sozialpsychologie 53, 4, 675-704

Glißmann, Wilfried, Klaus Peters (2001): Mehr Druck durch mehr Freiheit. Die neue Autonomie in der Arbeit und ihre paradoxen Folgen. Hamburg

Haipeter, Thomas (2005): Neue Steuerungsformen der Arbeit und ihre kollektivvertragliche Regulierung: Ansatzpunkte einer neuen Arena der industriellen Beziehungen?; in: Hilde Wagner (Hg.): „Rentier’ ich mich noch?“ Neue Steuerungskonzepte im Betrieb. Hamburg, 284-306

Haipeter, Thomas, Steffen Lehndorff (2004): Atmende Betriebe, atemlose Beschäftigte: Erfahrungen mit neuartigen Formen betrieblicher Arbeitszeitregulierung. Berlin

Haipeter, Thomas, Steffen Lehndorff (2002): Regulierte Flexibilität? Arbeitszeitregulierung in der deutschen Automobilindustrie; in: WSI-Mitteilungen 55, 5, 649-655.

Hartz, Peter (1996), Das atmende Unternehmen. Jeder Arbeitsplatz hat einen Kunden. Frankfurt/Main, New York

Höpner, Martin (2003), Wer beherrscht die Unternehmen? Shareholder Value, Managerherrschaft und Mitbestimmung in Deutschland, Frankfurt/Main, New York

Jackson, Gregory (2003), Corporate Governance in Germany and Japan: Liberalization Pressures and Responses during the 1990s; in: Kozo Yamamura, Wolfgang Streeck (eds.), The End of Diversity? Prospects for German and Japanese Capitalism. Ithaca, London: Cornell University Press, 261-305 
Jürgens, Ulrich, Frieder Naschold (Hg.) (1983): Arbeitspolitik. Materialien zum Zusammenhang von politischer Macht, Kontrolle und betrieblicher Organisation der Arbeit. Opladen

Kädtler, Jürgen (2003), Globalisierung und Finanzialisierung. Zur Entstehung eines neuen Begründungskontextes für ökonomisches Handeln; in: Klaus Dörre, Berndt Röttger (Hg.): Das neue Marktregime. Konturen eines nachfordistischen Produktionsmodells. Hamburg, 227-249

Kotthoff, Hermann (1994): Betriebsräte und Bürgerstatus. Wandel und Kontinuität betrieblicher Mitbestimmung. München, Mering

Kratzer, Nick, Dieter Sauer (2003): Andere Umstände - neue Verhältnisse: Ein Orientierungsversuch für Arbeitsforschung und Arbeitspolitik; in: WSI-Mitteilungen 56, 10, 578-583

Kuhlmann, Martin, Hans Joachim Sperling, Sonja Balzert (2004): Konzepte innovativer Arbeitspolitik. Good-Practice-Beispiele aus dem Maschinenbau, der Automobil-, Elektro- und Chemischen Industrie. Berlin

Moldaschl, Manfred (2001): Herrschaft durch Autonomie - Dezentralisierung und widersprüchliche Arbeitsanforderungen; in: Burkhard Lutz (Hg.): Entwicklungsperspektiven von Arbeit. Weinheim, 132-164

Sauer, Dieter (2005): Arbeit unter (Markt-)Druck: Ist noch Raum für innovative Arbeitspolitik?; in: WSIMitteilungen 58, 4, 179-185

Seifert, Hartmut (2001): Zeitkonten: Von der Normalarbeitszeit zu kontrollierter Flexibilität; in: WSIMitteilungen 54, 2, 84-90

Schmidt, Rudi, Rainer Trinczek (1999): Der Betriebsrat als Akteur der industriellen Beziehungen; in: Walther Müller-Jentsch (Hg.): Konfliktpartnerschaft. Akteure und Institutionen der industriellen Beziehungen. München/Mering (3. Aufl.), 103-128

Weltz, Friedrich (1988): Die doppelte Wirklichkeit der Unternehmen und ihre Konsequenzen für die Industriesoziologie; in: Soziale Welt 39, 97-103

Weltz, Friedrich (1977): Kooperative Konfliktverarbeitung - Ein Stil industrieller Beziehungen in deutschen Unternehmen; in: Gewerkschaftliche Monatshefte 5, 291-302

Yin, Robert K. (1988): Case Study Research. Design and Methods. Newbury Park u.a.O. (Rev. Ed.)

Anschrift des Verfassers:

Dr. Thomas Haipeter

Institut Arbeit und Technik (IAT) Gelsenkirchen

Schwerpunkt Arbeitszeit und Arbeitsorganisation

Munscheidstraße 14

D-45886 Gelsenkrichen

haipeter@iatge.de

Schlagwörter: Arbeitszeit, industrielle Beziehungen, Arbeitspolitik 